\title{
ANATOMIA DO ESCAPO FLORAL DE ESPÉCIES BRASILEIRAS DE PAEPALANTHUS SUBGÊNERO PLATYCAULON (ERIOCAULACEAE) ${ }^{1}$
}

\author{
Vera Lucia Scatena ${ }^{2}$ \\ Ana Maria Giulietti ${ }^{3}$ \\ Vanessa de Aquino Cardoso ${ }^{2}$
}

\section{Recebido em 28/01/97. Aceito em 07/05/1998}

\begin{abstract}
RESUMO - (Anatomia do escapo floral de espécies brasileiras de Paepalamthus subgênero Platycaulon (Eriocaulaceae). Foi estudada a anatomia dos escapos de 17 espécies de Paepalanthus subgênero Platycaulon, sendo 10 da sect. Divisi e sete da sect. Conferti. O trabalho foi realizado para caracterizar anatomicamente os escapos. como contribuição para o entendimento do grupo, uma vez que. morfologicamente, esse é o caráter taxonómico mais importante. Procurouse. também, confirmar ou não o reconhecimento das duas seções dentro do subgênero. Para os estudos anatômicos utilizou-se material proveniente do Brasil. obtido de exsicatas de diferentes herbários e/ou coletado na Serra do Cipó, MG. Neste trahalho observou-se que Paepalanthus subg. Platycaulon é caracterizado morfologicamente por apresentar escapos pluricapitulados no ápice. Anatomicamente, as espécies estudadas da sect. Divisi apresentam escapos com vários cilindros vasculares, na região mediana e. ainda apresentam. em Paepalanthus vellozioides e P. spixianus, feixes vasculares corticais, características únicas na família. Diferentemente, as espécies avaliadas da sect. Conferti apresentam escapos com cilindro vascular único na região mediana, padrão análogo ao das demais Eriocaulaceac. c ainda apresentam, em Paepalanthus itatiaiensis, P. planifolitus e P. paulensis, feixes vasculares medulares, que até então não haviam sido referidos para a familia.
\end{abstract}

Palavras-chave - Paepalanthus, anatomia. Platycaton

\begin{abstract}
Anatomy of the inflorescence scape of Brazilian species of the Paepalanthus subgenus Platycaulon (Eriocaulaceae). The scape anatomy of 17 taxa of Paepalanthus subgenus Platycaylon were studied, being 10 taxa of sect. Divisi and seven of sect. Conferti. The study was carried out to see whether scape morphology and anatomy provide valid taxonomic characters at the subgeneric level in Paepalamthus and to test a proposal to recognize two sections within Paepalanthus subgenus Platycaylon: sect. Divisi and sect. Conferti. The material for anatomical study was collected in Brazil. partly from herbarium specimens and partly from
\end{abstract}

Trabalho apresentado no XLVII Congresso Nacional de Botânica. Nova Friburgo, RJ, Brasil Departamento Botânica. Instituto de Biociências. UNESP. C. Postal 199. CEP 13506-900. Rio Claro. SP. Brasil. Bolsa CNPq

Departamento de Ciências Biológicas, Universidade Estadual de Feira de Santana, Km 3 BR 116. Campus Universitário. CEP 4403/-460. Feira de Santana. BA. Brasil. Bolsa CNPq 
fresh material collected in the field, from the Serra do Cipó. Minas Gerais State. In this work, we observe that Paepalanthus subg. Platycaulon is characterized morphologically by its pluricapitulate scapes. Anatomycally, the studied species of the Divisi sect. are characterized by having separate vascular cylinders, and present cortical vascular bundles in Paepalanthus vellozioides and $P$. spixianus, being these characteristics unique to the family, while the scapes of the studied members of sect. Conferti display a continuous vascular cylinder, which is analogous with other groups within the Eriocaulaceac, and present pith vascular bundles in Paepalanthus itatiaiensis, P. planifolius and P. paulensis. characteristics unique to the family.

Key words - Paepalanthus, anatomy, Platycaulon

\section{Introdução}

A família Eriocaulaceae é composta por cerca de 1.200 espécies, distribuídas em 10 gêneros, com distribuição pantropical. O gênero Eriocaulon inclui toda a área de distribuição geográfica da família; dentre os nove restantes, alguns têm áreas de distribuição menos amplas, como Mesanthemum, restrito à África, Lachnocaulon, às Américas do Norte e Central, Rondonanthus exclusivo da Venezuela e Guianas, além de Philodice e Blastocaulon, endêmicos de pequenas áreas do Brasil, em Mato Grosso e Minas Gerais, respectivamente. Os outros gêneros têm distribuição mais ampla, como Leiothrix e Tonina, ocorrentes em toda a área neotropical, e Syngonanthus e Paepalanthus, com distribuição disjunta na África e América do Sul (Giulietti \& Hensold 1990; 1991; Hensold \& Giulietti 1991).

Eriocaulon e Paepalanthus, em número de espécies, são os maiores gêneros da família, com cerca de 450 espécies cada um deles (Moldenke 1971). Eriocaulon reune especialmente plantas aquáticas marginais aos riachos mantendo, talvez por isso, certa uniformidade morfológica e anatômica (Monteiro et al. 1984). As espécies de Paepalanthus, por outro lado, podem ocorrer nos mais diversos habitats, incluindo desde solos arenosos úmidos ou secos até áreas desprovidas de solo, sobre pedras. Podem ser encontradas totalmente expostas ou com pouca exposição à luz, como nas bordas de cavernas e desde as restingas litorâneas até as montanhas andinas, com mais de $2.400 \mathrm{~m}$ de altitude. Tal variabilidade de habitat associada à grande diversidade não só de hábitos, com plantas de alguns milímetros até dois metros de altura, como das inflorescências, das flores (dímeras e trímeras) e dos frutos e sementes, tornam o gênero altamente polimórfico, tendo inclusive sido reconhecido recentemente como parafilético (Giulietti et al. 1995).

Ruhland (1903), na sua classificação de Paepalanthus, propõe a existência de cinco subgêneros: Paepalocephalus, Bostrychophyllum, Platycaulon, Xeractis e Thelxinö̈. Hensold \& Giulietti (1991) transferiram Paepalanthus subg. Bostrychophyllum para o gênero Rondonanthus

Paepalanthus subgen. Platycaulon Mart. é caracterizado por apresentar escapo pluricapitulado no ápice, capítulos com brácteas involucrais glabras internamente e espata truncada no ápice. Por esse conjunto de caracteres, Tissot-Squalli (1997) considera-o um grupo natural, com 46 espécies, das quais 31 ocorrem no Brasil, sendo 19 em Paepalanthus sect. Divisi Ruhland e 12 em Paepalanthus sect. Conferti Ruhland, com distribuição disjunta no Brasil, países Andinos e na Costa Rica. 
Os estudos da estrutura anatômica do escapo em Eriocaulaceae têm recebido menos atenção do que a estrutura anatômica da folha, provavelmente devido à maior uniformidade dos escapos na família. Malmanche (1919) e Tomlinson (1969) consideraram que os escapos possuem estrutura radiada formada por regiões do córtex que constituem as costelas (tecido de sustentação) e por regiões intercostais (parênquima clorofiliano). A morfoanatomia dos escapos florais em Eriocaulaceae tem sido usada com fins taxonômicos e em diferentes níveis hierárquicos. Segundo Tomlinson (1969), o gênero Eriocaulon apresenta epítetos específicos que são relacionados com o número de costelas do escapo (por exemplo, E. septangulare, E. decangulare, E. quinquangulare). Para o gênero Syngonanthus, Scatena \& Menezes (1996) relatam que o número de costelas dos escapos pode auxiliar na separação das seções dentro do gênero. No gênero Leiothrix, no entanto, pode ocorrer variação no número de costelas, dentro da mesma espécie e inclusive do mesmo indivíduo, como foi demonstrado por Scatena \& Giulietti (1996), em populações de Leiothrix crassifolia e em L. flavescens (Scatena et al. 1998).

Segundo Malmanche (1918) e Tomlinson (1969), de todos os escapos por eles estudados, apenas Paepalanthus spixianus apresenta simetria bilateral. No gênero Paepalanthus a anatomia dos escapos de espécies de Paepalanthus subg. Platycaulon só foi estudada para P. spixianus por Malmanche (1919) e Tomlinson (1969) e, para outras cinco espécies, por Scatena \& Moraes (1996), sendo três pertencentes à sect. Divisi e duas à sect. Conferti.

O objetivo deste trabalho foi caracterizar anatomicamente os escapos de Paepalanthus subg. Platycaulon como uma contribuição para o entendimento do grupo, uma vez que morfologicamente esse é o caráter taxonômico considerado mais importante. Também procurou-se contribuir com dados relevantes para a verificação da validade dos grupos infragenéricos propostos por Ruhland (1903), Paepalanthus subg. Platycaulon sect. Divisi e sect. Conferti.

\section{Material e métodos}

O material estudado de Paepalanthus subg. Platycaulon, proveniente de diferentes regiões do Sudeste e Sul do Brasil, teve as identificações atualizadas segundo Tissot-Squalli (1997) e são apresentados de acordo com a seção correspondente:

\section{Paepalanthus subg. Platycaulon Mart. sect. Divisi Ruhland}

\section{Paepalanthus albo-vaginatus Silveira var. fuscobracteatus Tissot-Squalli}

Material examinado: BRASIL. Paraná: Balsa Nova, Alto do Purunã, Hatschbach 42657 (SPF). P. bromelioides Silveira. Minas Gerais: Santana do Riacho, Serra do Cipó, Sano et al. CFSC 12859 (SPF); Scatena s.n. (BHCB 20672). P. corymbosus (Bong.) Kunth.; Tiradentes, Serra de São José, Alves \& Kolbek 752 (SPF). P. hydra Ruhland.; Lavras Novas, Giulietti et al. CFCR 9199 (SPF). P. latipes Silveira; Santana do Riacho, Serra do Cipó, Tissot \& Scatena 29 (BHCB). P. longicaulis Silveira; Serra do Cipó, Silveira 692 (R). P. melaleucus (Bong.) Kunth; Serra do Caraça, Silveira 431 (R). P. 
moedensis Silveira; Serra da Moeda, Silveira 572 (R). P. spixianus Mart.; Caeté, Serra da Piedade, Grandi et al. 1890 (SPF). P. trichopetalum Mart.; Diamantina, Pereira 2839 \& Pabst 3675 (HB). P. vellozioides KoernBrumadinho, Martens s.n. (SPF 87339).

\section{Paepalanthus subg. Platycaulon Mart. sect. Conferti Ruhland}

\section{Paepalanthus conduplicatus Koern. var. pubescens Silveira}

Material examinado: BRASIL. Minas Gerais: Serra do Cipó, Silveira 555 (R). P. globulifer Silveira; Serra do Cipó, Giulietti \& Menezes CFSC 3979 (SPF). P. itatiaiensis Ruhland.; Serra de Ibitipoca, Silveira 599 (R); Santana do Riacho, Serra do Cipó, BenkoIseppon s.n. (SPF 94431). P. paulensis Ruhland.; Datas, Morro do Coco, Mello-Silva et al. CFCR 8023 (SPF); Santana do Riacho, Serra do Cipó, Scatena \& Tissot 35 (BHCB), Scatena s.n. (BHCB 27826). Rio de Janeiro: Serra de Itatiaia, Silveira 607 (R). P. macropodus Ruhland.; São Paulo: Serra da Bocaina, Schwacke 1936 (RB). P. planifolius Koern.

O estudo anatômico dos escapos adultos das espécies de Paepalanthus subgêneros Platycaulon foi feito em material herborizado, fervido em água, com gotas de glicerina para expansão dos tecidos, e, posteriormente, transferido para álcool etílico $70 \%$. Fazem exceção $P$. bromelioides e $P$. planifolius cujos escapos jovens e adultos foram coletados e fixados em formol 5 partes: ácido acético glacial 5 partes: etanol $50 \%$ 90 partes (Johansen 1940). As secções anatômicas foram feitas à mão livre, com auxílio de lâmina de barbear, na região mediana dos escapos. Em P. bromelioides e P. planifolius foram obtidas secções nos diferentes níveis dos escapos jovens e adultos. As secções foram coradas com fucsina e azul de astra (Roeser 1962) e montadas em gelatina glicerinada entre lâmina e lamínula.

Os desenhos da morfologia externa dos escapos de P. bromelioides e P. planifolius foram feitos à mão livre, diretamente dos materiais herborizados e fixados. Os desenhos anatômicos esquemáticos foram realizados ao microscópio de luz com o emprego de câmara-clara e as fotos foram realizadas ao fotomicroscópio (Zeiss), ambos com projeção da escala micrométrica.

\section{Resultados e discussão}

As espécies de Paepalanthus subg. Platycaulon estudadas apresentam os escapos medindo de 20 a $80 \mathrm{~cm}$ alt. Os representantes de Paepalanthus subg. Platycaulon sect. Divisi caracterizam-se por apresentarem os escapos divididos na região apical, junto às inflorescências (Fig. 1-2 setas) e aqueles de Paepalanthus subg. Platycaulon sect. Conferti caracterizam-se por não apresentarem os escapos assim divididos (Fig. 8-9 setas).

Os diagramas das Fig. 3-7 correspondem às secções transversais feitas em diferentes níveis no escapo jovem de P. bromelioides (sect. Divisi) (Fig. 2) e representam os padrões anatômicos dos mesmos. O escapo de $P$. bromelioides é constituído por epiderme, córtex e cilindro vascular. O córtex é constituído por tecido de sustentação mais parênquima clorofiliano e endoderme que delimita o cilindro vascular, que é 


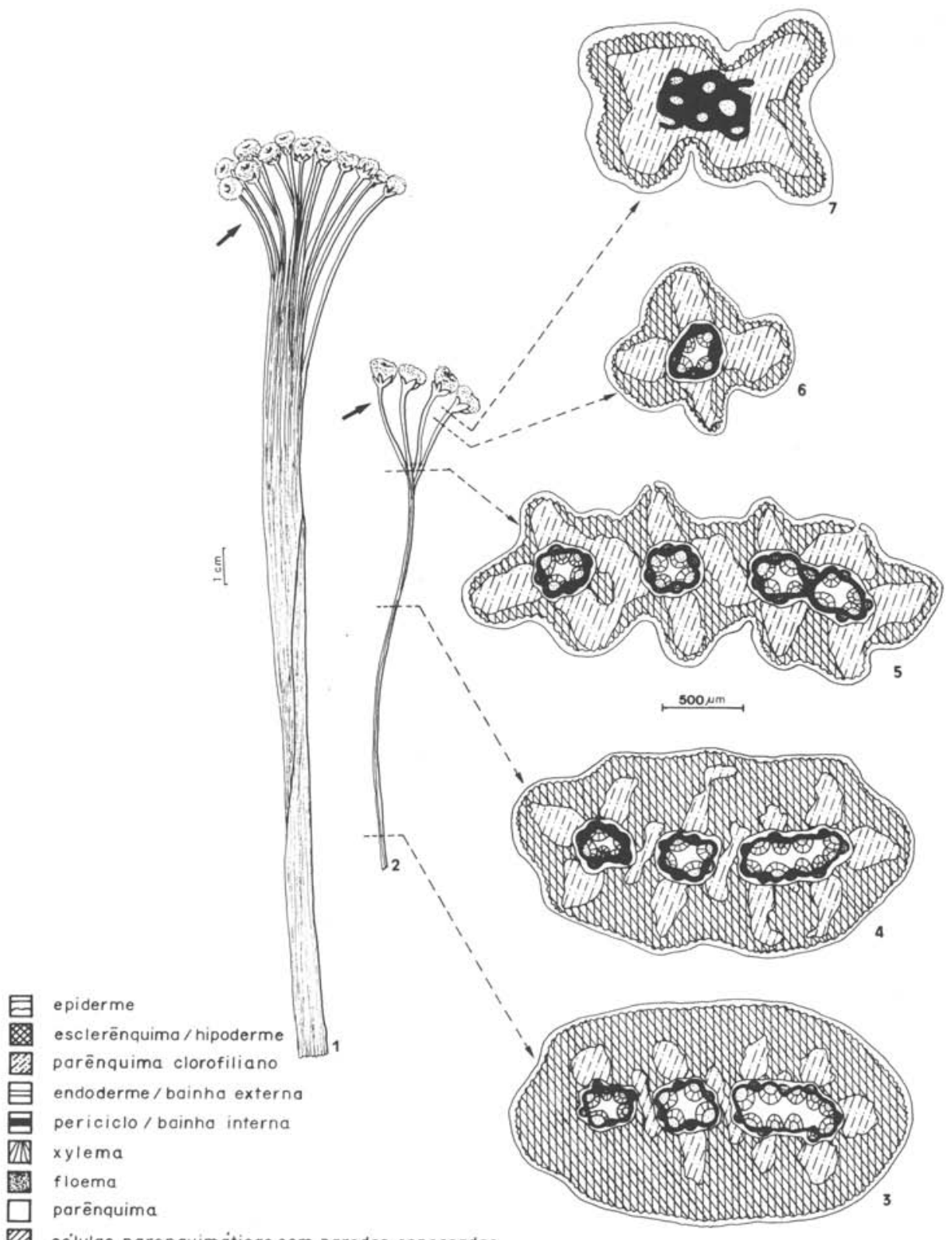

Células parenquimáticas com paredes espessadas

a. feixes vosculares

Figuras 1-7. Morfologia e anatomia do escapo de P. bromelioides (sect. Divisi). 1. Escapo adulto. 2. Escapo jovem. 3-7. Esquemas das secções transversais do escapo jovem de $\boldsymbol{P}$. bromelioides nas regiōes: 3 . basal, 4. mediana e 5-7. apicais. 


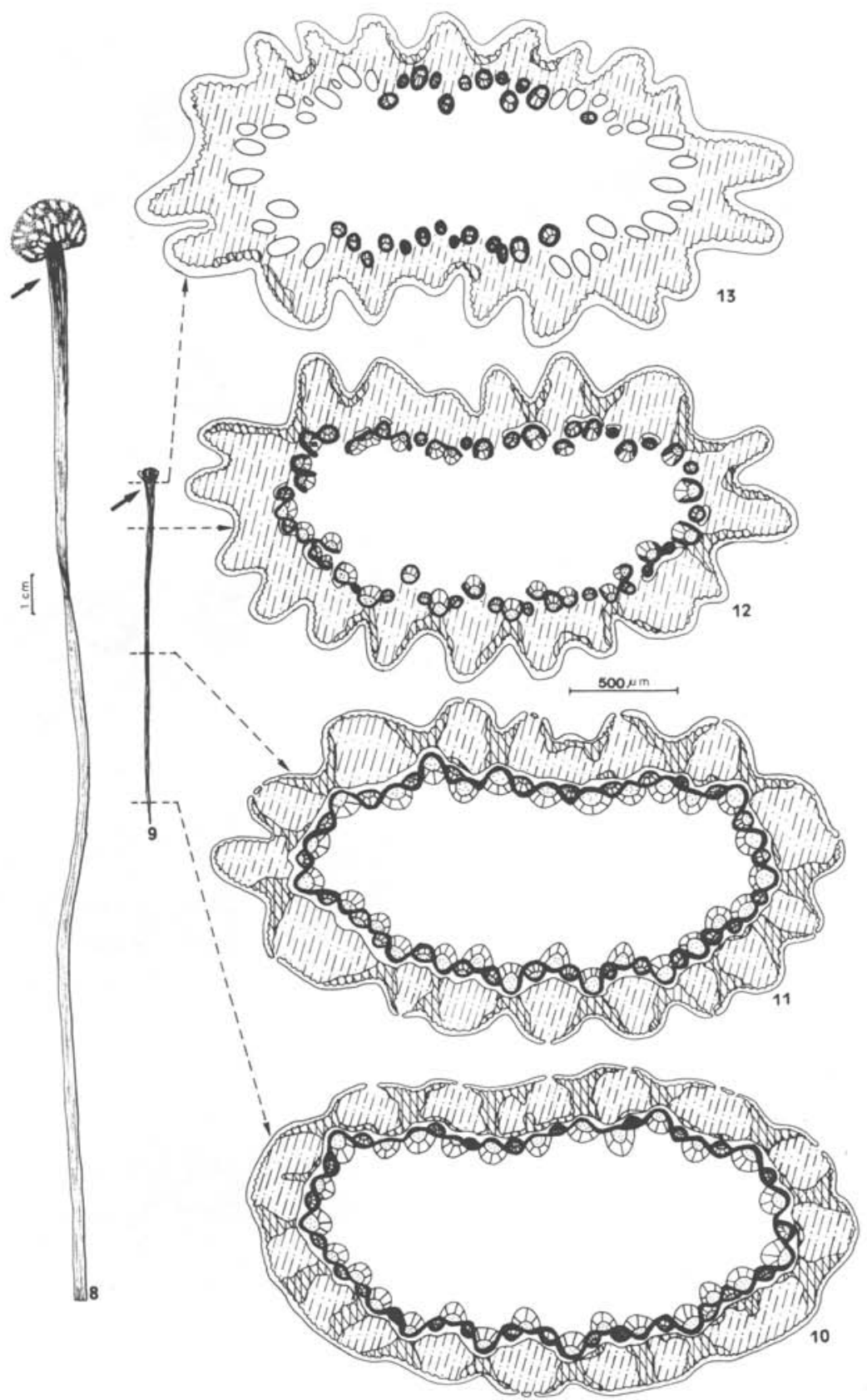

Figuras 8-13. Morfologia e anatomia do escapo de P. planifolius (sect. Conferti). 8. Escapo adulto. 9. Escapo jovem. 10-13. Esquemas das secções transversais do escapo jovem de $\boldsymbol{P}$. planifolius nas regiôes: 10 . basal, 11. mediana e 12-13. apicais. 
constituído pelo periciclo mais os feixes vasculares e a medula. Nas regiões basal (Fig. 3) e mediana (Fig. 4), observam-se três cilindros vasculares, sendo que o da direita possui o dobro do tamanho dos demais. Na região apical (Fig. 5) observa-se que o cilindro vascular da direita (maior) apresenta-se dividido em dois, estabelecendo assim quatro divisões externas, que constituem os escapos divididos que sustentam os capítulos no seu ápice. Já na Fig. 6 observa-se uma dessas divisões isoladamente e, na Fig. 7, observa-se a orientação dos feixes vasculares periféricos que irão para as brácteas que envolvem o capítulo. Cada escapo apical possui o mesmo padrão anatômico das demais regiões (Fig. 3-7), apresentando-se isolado no ápice (Fig. 6) e unido na região basal (Fig. 3).

Os diagramas das Fig. 10-13 correspondem às secções transversais feitas em diferentes níveis no escapo jovem de P. planifolius (sect. Conferti) (Fig. 9) e representam os padrões anatômicos dos mesmos. O escapo de $P$. planifolius é constituído de epiderme, córtex e cilindro vascular com distribuição e constituição similares às já descritas para P. bromelioides. Nas regiões basal (Fig. 10), mediana (Fig. 11) e apical (Fig. 12-13) o cilindro vascular não se divide interna e nem externamente.

Os diagramas das secções transversais dos escapos da região mediana de todas as espécies estudadas de Paepalanthus subg. Platycaulon podem ser observados nas Fig. 14-30. Todos eles possuem forma achatada ou oval, com simetria bilateral. Os escapos das espécies de Paepalanthus sect. Divisi estão representados nas Fig. 14-23. P. bromelioides, $P$. corymbosus, $P$. hydra, $P$. latipes, $P$. vellozioides e $P$. longicaulis apresentam o periciclo dividido, formando cilindros vasculares distintos (Fig. 14-18). Paepalanthus melaleucus, P. trichopetalum, P. moedensis, P. spixianus e P. albovaginatus var. fuscobracteatus apresentam o cilindro vascular único, não dividido (Fig. 19-23). Na região apical, todas as espécies apresentam os escapos divididos, com os capítulos na porção terminal, como pode ser observado em P. bromelioides (Fig. 1-2). Os escapos das espécies de Paepalanthus sect. Conferti apresentam cilindro vascular único (Fig. 24-30). Na região apical, todos os escapos apresentam-se indivisos com os capítulos unidos entre si, adquirindo a forma globóide, como pode ser observado em $P$. planifolius (Fig. 8-9).

Em todas as espécies estudadas, a epiderme (E) é unisseriada e pode apresentarse com células totalmente espessadas, como em P. bromelioides (Fig. 31), P. corymbosus, P. vellozioides, P. trichopetalum, P. moedensis, P. spixianus, P. itatiaiensis, P. globulifer; P. macropodus, P. paulensis, P. longicaulis e P. conduplicatus var. pubescens (Fig. 34). Também pode apresentar-se com células parcialmente espessadas, como em $P$. hydra (Fig. 32), P. melaleucus, P. latipes, P. albo-vaginatus var. fucsobracteatus, P. itatiaiensis e P. planifolius (Fig. 33).

O córtex é constituído de parênquima clorofiliano associado ao tecido de sustentação que forma as costelas (Fig. 31-34). As costelas podem ser salientes, como em P. melaleucus, P. trichopetalum, P. spixianus, P. itatiaiensis, P. conduplicatus var. pubescens, P. globulifer; P. paulensis e P. planifolius (Fig. 33). O córtex pode apresentarse com costelas não salientes, com em $P$. bromelioides (Fig. 31), P. hydra (Fig. 32), $P$. corymbosus, P. latipes, $P$. vellozioides, P. moedensis, P. albo-vaginatus var. fuscobracteatus, P. itatiaiensis, P. macropodus e P. longicaulis. 


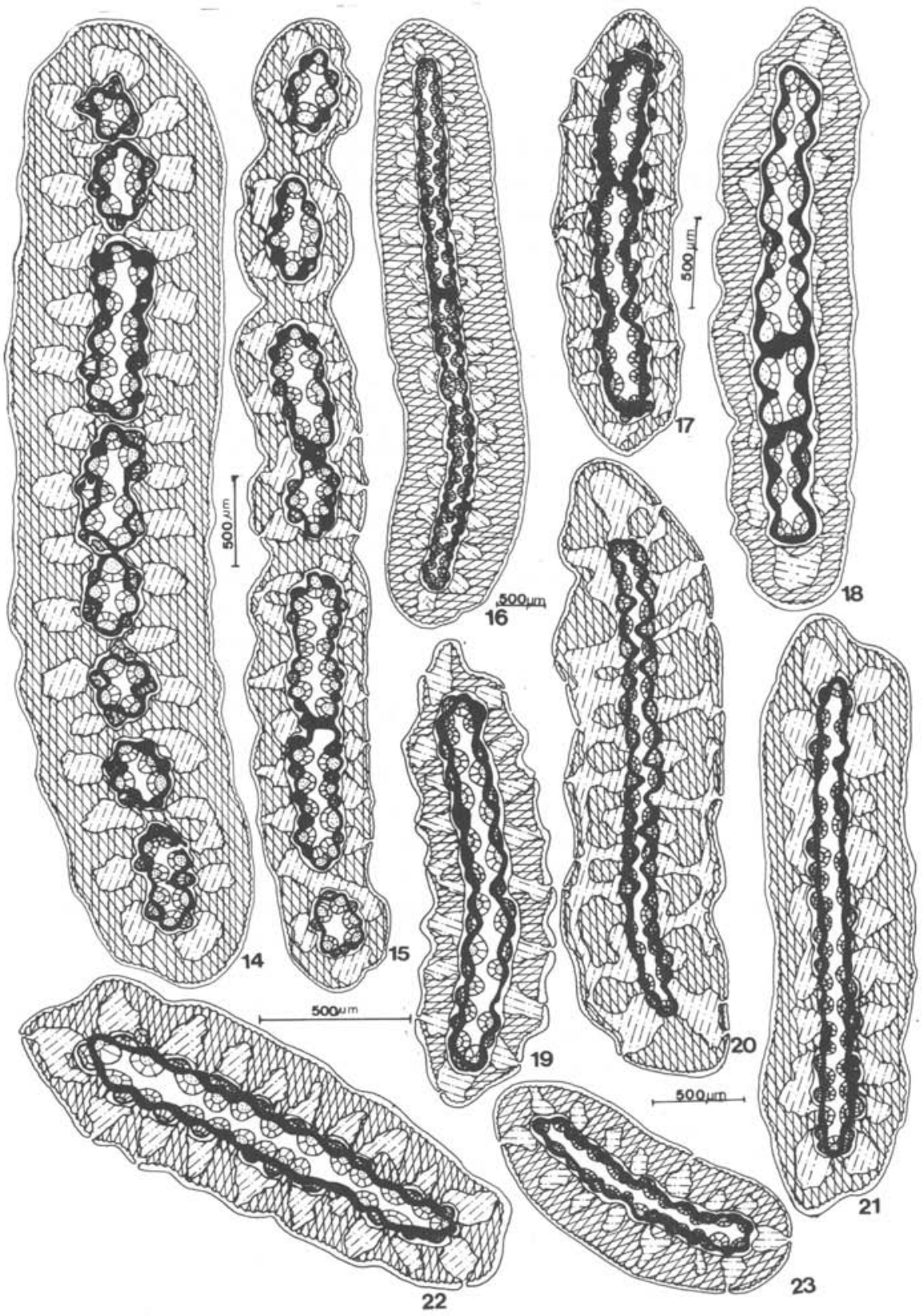

Figuras 14-23. Esquemas das secções transversais na região mediana dos escapos de Paepalanthus subg. Platycaulon sect. Divisi. 14. P. bromelioides. 15. P. hydra. 16. P. latipes. 17. P. vellozioides. 18. P. longicaulis. 19. P. melaleucus. 20. P. trichopetalum. 21. P. moedensis. 22. P. spixianus. 23. P. albo-vaginatus var. fuscobracteatus. 

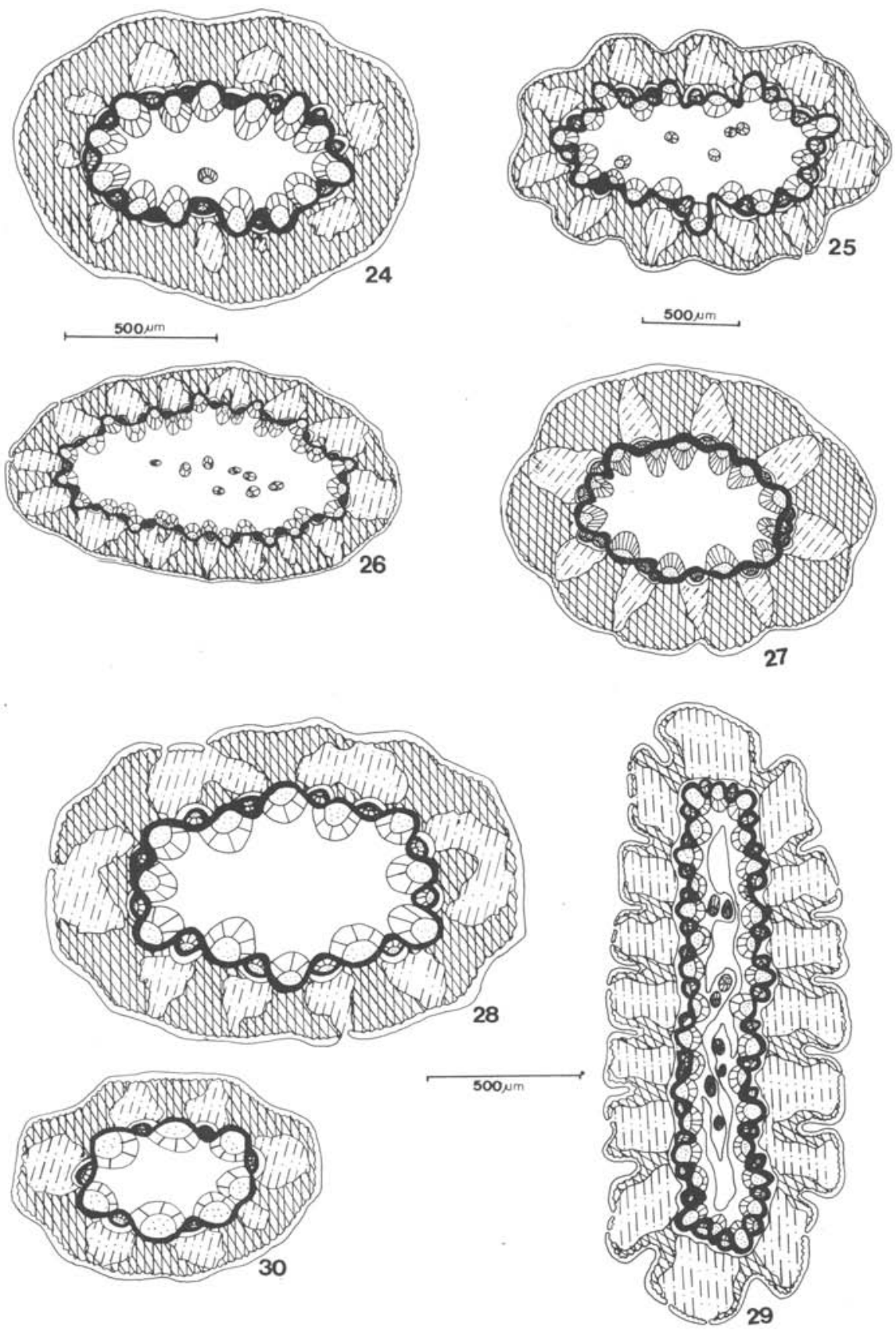

Figuras 24-30. Esquemas das secções transversais na região mediana dos escapos de Paepalanthus subg. Platycaulon sect. Conferti. 24-25. P. itatiaiensis. 26. P. planifolius. 27. P. globulifer. 28. P. macropodus. 29. P. paulensis. 30. P. conduplicatus var. pubescens. 
No córtex, as células do tecido de sustentação situadas próximas à epiderme são esclerenquimáticas na maioria das espécies (Fig. 31-32,34), enquanto que aquelas células mais próximas do cilindro vascular são colenquimáticas (Fig. 31-32,34). Além das características já referidas, foi observada a presença de feixes vasculares corticais em $P$. vellozioides (Fig. 17) e P. spixianus (Fig. 22), ambos de Paepalanthus sect. Divisi.

Neste trabalho, a continuidade da endoderme foi definida quando este tecido envolve totalmente o cilindro vascular, independente da divisão ou não dos escapos no seu ápice. A descontinuidade da endoderme está associada à interrupção da mesma, na altura dos tecidos de sustentação presentes nas costelas. A endoderme (EN) pode apresentar-se contínua, como em P. bromelioides (Fig. 14,31), P. latipes, P. hydra (Fig. 15, 32), P. corymbosus, P. vellozioides, P. melaleucus, $P$. albo-vaginatus var. fuscobracteatus, $P$. paulensis e $P$. longicaulis. A endoderme pode apresentar-se descontínua, como em P. trichopetalum, P. moedensis, P. spixianus, P. itatiaiensis, P. planifolius (Fig. 26, 33), P. macropodus, P. globulifer e P. conduplicatus var. pubescens (Fig. 30, 34).

O cilindro vascular possui o periciclo $(\mathrm{P})$ de forma sinuosa, envolvendo totalmente os feixes menores e formando calota nos maiores. As células do periciclo normalmente possuem a parede totalmente espessada. Na região apical do escapo, o periciclo apresenta-se dividido, formando cilindros vasculares isolados em todas as espécies de Paepalanthus sect. Divisi, e não dividido em todas as espécies de Paepalanthus sect. Conferti. Na região mediana dos escapos de algumas espécies de Paepalanthus sect. Divisi, o periciclo não está dividido, como em $P$. melaleucus (Fig. 19), P. trichopetalum (Fig. 20), P. moedensis (Fig. 21), P. spixianus (Fig. 22) e P. albo-vaginatus var. fuseobracteatus (Fig. 23). As espécies restantes, P. bromelioides (Figs. 14, 31), P. corymbosus, P. hydra (Fig. 15), P. latipes (Fig. 16), P. vellozioides (Fig. 17) e P. longicaulis (Fig. 18), apresentam o periciclo já dividido nessa região.

Em todas as espécies estudadas os feixes vasculares são colaterais e a medula é constituída por células de parênquima de parede fina (Fig. 33-34). Paepalanthus itatiaiensis (Fig. 24-25), P. planifolius (Fig. 26) e P. paulensis (Fig. 29), pertencentes à sect. Conferti, possuem feixes vasculares na medula com números variados.

A maioria das espécies estudadas apresenta as células epidérmicas dos escapos com a parede totalmente espessada, porém algumas apresentam-na parcialmente espessada. Essa característica foi observada, também, nos escapos de espécies de Syngonanthus (Scatena \& Menezes 1996) e nos escapos de várias populações de Leiothrix crassifolia (Scatena \& Giulietti 1996). Além dos espessamentos das células da epiderme, os escapos apresentam esclerênquima logo abaixo da epiderme, que, juntamente com o parênquima clorofiliano, formam as costelas dos mesmos. A presença de epiderme com parede espessada associada ao tecido de sustentação permite que os escapos fiquem enrijecidos e, assim, sustentem os capítulos no seu ápice, característica imprescindível em Paepalanthus subg. Platycaulon, onde houve a junção de vários capítulos, acarretando maior peso sustentado por um único eixo.

O número de costelas dos escapos é frequentemente usado como caráter taxonômico ao nível de espécie para as Eriocaulaceae (Tomlinson 1969; Giulietti 1984). 

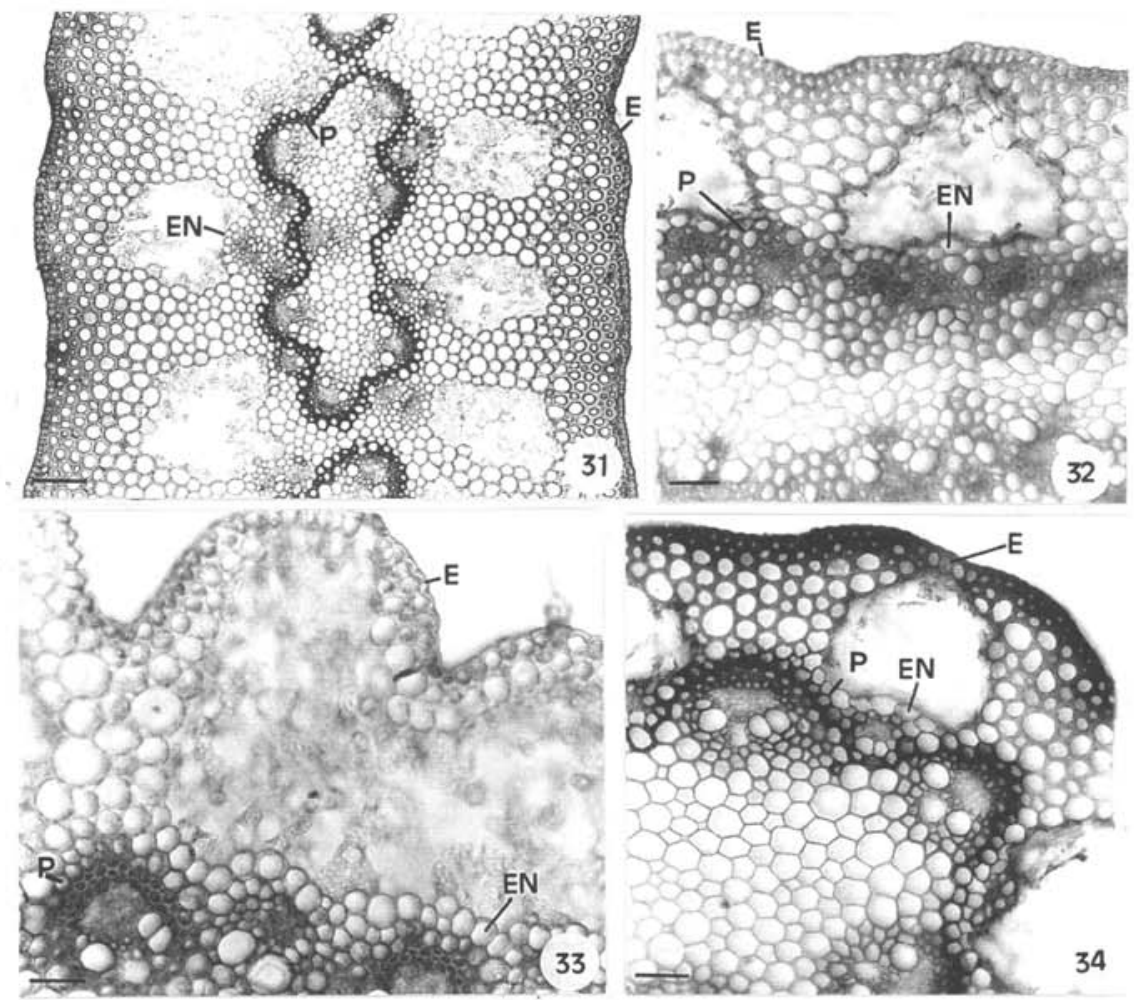

Figuras 31-34. Secções transversais da região mediana dos escapos de Paepalanthus subgênero Platycaulon. Sect. Divisi: 31 . P. bromelioides $($ Barra $=100 \mathrm{~mm})$ e 32 . P. hydra $($ Barra $=50 \mathrm{~mm})$. Sect Conferti: 33. P. planifolius $(\mathrm{Barra}=50 \mathrm{~mm})$ e 34. $P$. conduplicatus var. pubescens $(\mathrm{Barra}=$ $50 \mathrm{~mm})$. (E - epiderme, EN - endoderme, $\mathrm{P}$ - periciclo).

Segundo Tomlinson (1969), os escapos possuem feixes vasculares maiores, que são os medulares, e estão para dentro da endoderme, e feixes vasculares menores, que são os corticais, e estão para fora da endoderme. O que o autor considerou endoderme, foi demonstrado por Scatena \& Menezes (1996) tratar-se do periciclo e, portanto, o cilindro vascular, com feixes vasculares maiores e menores, apresenta-se delimitado pela endoderme.

A presença de feixes vasculares corticais nos escapos de vários gêneros de Eriocaulaceae tem sido referida por Malmanche (1919), Tomlinson (1969), Giulietti (1984) e Hensold (1988). Os autores denominam feixes vasculares corticais aqueles externos à endoderme. Nesse trabalho, tais feixes foram considerados como feixes vasculares menores do estelo dos escapos, estando, portanto, ocupando uma posição interna à endoderme. Externamente ao periciclo ocorre uma camada de células parenquimáticas, a endoderme, que pode ser contínua, formando um cilindro, ou descontínua, formando calotas sobre os feixes vasculares menores. Em Paepalanthus vellozioides $\mathrm{e}$ P. spixianus 
de Paepalanthus subg. Platycaulon sect. Divisi ocorrem feixes vasculares corticais, situados para fora da endoderme, sendo esta a primeira referência dessa característica para a família. Em $P$. itaticiensis, $P$. planifolius e $P$. paulensis de Paepalanthus subg. Platycaulon sect. Conferti ocorrem feixes vasculares medulares. Tal característica também não havia sido referida para a família.

Nas espécies estudadas foram observados feixes vasculares corticais em algumas espécies de Paepalanthus subg. Platycaulon sect. Divisi e feixes vasculares medulares em algumas espécies de Paepalanthus subg. Platycaulon sect. Conferti que podem ser utilizadas como características diferenciais. Mesmo ocorrendo apenas em algumas das espécies estudadas, podem ser utilizadas na distinção de Paepalanthus subg. Platycaulon sect. Divisi e sect. Conferti.

O tamanho, a forma em secção transversal do escapo e o número de costelas são caracteres taxonômicos muito utilizados ao nível específico em Syngonanthus (Scatena \& Menezes 1996), Leiothrix (Giulietti 1984; Scatena \& Giulietti 1996; Scatena et al. 1998) e Paepalanthus subg. Xeractis (Hensold 1988). Os escapos, que sustentam um só capítulo no seu ápice, apresentam número reduzido de feixes vasculares menores e maiores no cilindro vascular central, facilitando a delimitação das costelas, que geralmente ficam salientes. Nas espécies de Paepalanthus subg. Platycaulon estudadas neste trabalho, os eixos dos escapos, como resultado da fusão de vários deles, são largos e, para a maioria das espécies, as costelas não são salientes.

Na sect. Divisi todos os escapos possuem simetria bilateral em corte transversal. Tal fato só tinha sido referido para a família por Tomlinson (1969) e Malmanche (1919) para P. spixianus, única espécie de Paepalanthus subg. Platycaulon estudada por esses autores. Na sect. Conferti a simetria dos escapos é também bilateral, apesar dos mesmos terem forma mais oval do que achatada, em corte transversal.

Com base nos resultados apresentados no presente trabalho, a característica anatômica que se mostrou mais significativa, portanto, para separar as espécies estudadas de Paepalanthus subg. Platycaulon sect. Divisi da sect. Conferti é, no primeiro táxon, a divisão do periciclo, formando vários cilindros vasculares na região apical dos escapos, sendo que essa característica já está presente em algumas espécies, mesmo na região mediana. Tal característica anatômica traduz-se morfologicamente pela divisão dos escapos no ápice. Nas espécies estudadas de Paepalanthus subg. Platycaulon sect. Conferti não ocorre divisão do periciclo e nem divisão do escapo. Ruhland (1903) utilizou esta característica morfológica para separar as seções dentro desse subgênero. A presença, nas espécies estudadas, de feixes vasculares no córtex em Paepalanthus subg. Platycaulon sect. Divisi e a presença de feixes vasculares na medula em Paepalanthus subg. Platycaulon sect. Conferti, são consistentes para ajudar na separação dos dois táxons, confirmando a proposta de Ruhland (1903).

Com base na morfologia e na anatomia dos escapos das espécies estudadas de Paepalanthus subg. Platycaulon, considera-se que o ocorrido foi a fusão de vários escapos isolados, para a formação dos escapos coletivos, ovais ou achatados, com divisão somente no ápice, em corte transversal. Provavelmente a sect. Conferti com escapo cilíndrico seja a mais evoluída, corroborando a proposta feita nessa mesma direção por Tissot-Squalli (1997). 


\section{Agradecimentos}

À Dra. Margarida Venturelli (in memorian) por ter iniciado estudos anatômicos neste grupo; ao Dr. Raymond M. Harley, pela correção do Abstract; à Carmem S.C. Ragghiante e à Célia M. Hebling, pela digitação; ao Sr. Luiz R. Jordão, pelo auxílio nas ilustrações.

\section{Referências bibliográficas}

Giulietti. A. M. 1984. Estudos taxonômicos do gênero Leiothrix Ruhl. (Eriocaulaceae). Tese de Livre Docência. Universidade de São Paulo. São Paulo.

Giulietti, A. M.; Amaral. M. C. E. \& Bittrich. V. 1995. Phylogenetic analysis of inter-and infrageneric relationships of Leiothrix Ruhland (Eriocaulaceac). Kew Bulletim 50(1): 55-71.

Giulieuti, A. M. \& Hensold. N. 1990. Padrões de distribuição geográfica dos gêneros de Eriocaulaceac. Acta Botanica Brasilica 4(1): 133-159.

Giulictti. A. M. \& Hensold. N. 1991. Synonimization of the genera Comanthera and Carptotepala with Singonanthus (Eriocaulaceae). Annals of the Missouri Botanical Garden 78: 273-295.

Hensold, N. 1988. Morphology and systematics of Paepalanthus subgenus Xeractis (Eriocaulaceae). Sistematic Botany Monographs. Michigan: The American Society of Plants Taxonomists. 23.

Hensold. N. \& Giulietti. A. M. 1991. Revision and redefinition of the genus Rondonanthus Herzog (Eriocaulaceae). Annals of the Missouri Botanical Garden 78: 441-459.

Johansen. D. A. 1940. Plant microthechnique. Mc Grall-Hill Book Co. New York.

Malmanche, L. A. 1919. Contribution à I'étude anatomique des Eriocaulonacées et des familles voisines. Restiacées, Centrolepidacées, Xyridacées, Philhydracées, Mayacacées. Thesis. Paris.

Moldenke, H. N. 1971. A fifth summary of the Verbenaceae, Avicenniaceae, Stilbaceae, Dicrastylidaceae, Symphoremaceae, Nyctanthaceae and Eriocaulaceae of the world as to valid taxons, geographic distribution and synonymy. 2 vols. Ann. Arbor. Braun-Brumfield. Michigan.

Monteiro. W. R.; Giulietti. A. M. \& Castro. M. M. 1984. Aspects of leaf structure of some species. of Eriocaulon L. (Eriocaulaceae) from Serra do Cipó (Minas Gerais. Brazil). Revista Brasileira de Botânica 7(2): 137-147.

Roeser. K. R. 1962. Die Nadel der Schwarzkiefer-Massenprodukt und Kunstwert der Natur. Mikrokosmos 61: 33-36.

Ruhland, W. 1903. Eriocaulaceae. In Engler. A. (Ed.). Das Pflanzenreich. IV. 30: 1-294. Wilhelm Engelmann. Leipzig.

Scatena, V. L. \& Giulietti. A.M. 1996. The taxonomy and morphological and anatomical differentiation of populations of Leiothrix crassifolia (Eriocaulaceac). Plant Systematics and Evolution 199: $243-258$.

Scatena, V. L. \& Menezes, N. L. 1996. Anatomia de escapos e folhas de Syngonanthus Ruhl. (Eriocaulaceae) de campos rupestres. Revista Brasileira de Biologia 56(2): 317-332.

Scatena. V. L. \& Moraes. A. R. S. 1996. Anatomia de raizes. folhas e escapos de Paepalanthus subgênero Platycaulon (Eriocaulaceae) da Serra do Cipó-MG. Arquivos de Biologia e Tecnologia 39(4): 1021-1035.

Scatena. V. L.; Cardoso. V. A. \& Giulietti, A. M. 1998. Anatomia de escapos c folhas c sua aplicação na taxonomia de Leiothrix flavescens (Bong.) Ruhl. (Eriocaulaceae). Sitientibus (no prelo).

Tissot-Squalli. M. L. 1997. Monographische Bearbeitung von Paepalanthus subgenus Platycaulon. Dissertationes Botanicae Band 280. J. Cramer. Stuttgart.

Tomlinson. P. B. 1969. Anatomy of the Monocotyledons III. Commelinales-Zingiberales. Clarendon Press.Oxford. 